\title{
Volume, funções probabilísticas e produtividade em plantio de Calophyllum brasiliense no município de Dueré (TO)
}

\author{
Marcos Cardoso Silva ${ }^{*}$ Amanda Correia Vieira ${ }^{1}$ Yandro Brigida Ataíde ${ }^{1}$ Yasmim Andrade Ramos ${ }^{1}$ Maria \\ Cristina Bueno Coelho ${ }^{1}$ Marcos Giongo ${ }^{1}$ Mauro Luiz Erpen²
}

${ }^{1}$ Universidade Federal do Tocantins, campus Gurupi, Chácara 69-72 Rua Badejos, Lote 7 s/n - Jardim Sevilha, Gurupi - TO, $77404-970$

${ }^{2}$ Instituto Federal do Tocantins, Alameda Madrid, 545 - Jardim Sevilha, Gurupi - TO, 77410-470

*Author for correspondence: markuz@uft.edu.br

Received: October 2018 / Accepted: March 2019 / Published: June 2019

\section{Resumo}

Os objetivos desse trabalho foram ajustar equações volumétricas bem como testar funções de densidade de probabilidade em plantio comercial de Calophyllum brasiliense aos 94 meses de idade (no ano de 2016), no município de Dueré (TO) com o objetivo de determinar a produtividade para dar suporte aos planos de manejo. A função descrita por Schumacher-Hall, foi a que melhor se ajustou ao conjunto de dados, apresentando: $\mathrm{R}^{2}$ aj de 0.979 e coeficiente de variação de $8,31 \%$. Com a equação selecionada estimou-se o volume de $92,3 \mathrm{~m}^{3} \mathrm{ha}^{-1} \mathrm{com}$ incremento médio anual em volume de $11,78 \mathrm{~m}^{3} \mathrm{ha}^{-1}$ ao ano, aos 94 meses, seu incremento periódico para a mesma idade foi de $40,38 \mathrm{~m}^{3} \mathrm{ha}^{-1}$ ao ano. A frequência dos indivíduos por classes de diâmetro foi estimada pela função probabilística por ter os melhores ajustes. Os resultados de crescimento, incremento e produtividade mostraram que a espécie é promissora e pode ser usada em plantios comerciais.

Palavras chaves: Plantios florestais, crescimento, espécies nativas, inventário florestal

\begin{abstract}
The objectives of this work were to adjust volumetric functions as well as to test probability density functions in commercial plantation of Calophyllum brasiliense at 94 months of age (in 2016), in the municipality of Dueré (TO) with the objective of determining productivity to support management plans. The function described by Schumacher-Hall was the one that best fit the data set, presenting: $\mathrm{R}^{2}$ aj of 0.979 and coefficient of variation of $8.31 \%$. With the selected equation, it was estimated the volume of $92.3 \mathrm{~m}^{3} \cdot \mathrm{ha}^{-1}$ with an annual average increase in volume of $11.78 \mathrm{~m}^{3} \cdot \mathrm{ha}^{-1}$ per year, at 94 months, its periodic increment for the same age was $40.38 \mathrm{~m}^{3} \cdot \mathrm{ha}^{-1}$ per year. The frequency of individuals by diameter classes was estimated by the probabilistic function to have the best adjustments. The results of growth, increase and productivity have shown that the species is promising and can be used in commercial plantations.
\end{abstract}

Keywords: Forest plantings, growth, native species, forest inventory

\section{Introdução}

Calophyllum brasiliense, mais conhecido como Guanandi, ou Landi, é uma espécie arbórea que ocorre naturalmente desde América Central $\left(18^{\circ} \mathrm{N}\right)$ até a América do Sul $\left(28^{\circ} 10^{\prime} \mathrm{S}\right)$. No Brasil, a espécie é encontrada na Amazônia, no Cerrado e na Mata Atlântica, desde o Estado do Amazonas até Santa Catarina (Morandi et al, 2017). Quando adulto, o Calophyllum brasiliense pode possuir de 40 a $45 \mathrm{~m}$ de altura, alcançando até $180 \mathrm{~cm}$ de diâmetro, sua copa é redonda e com folhas verde escuras, os galhos são obliquamente ascendentes, seu fuste é cilíndrico e com base cônica (Busato et al, 2016). No Brasil, tem alto valor econômico pela larga utilização para os mais diversos fins, tais como construção civil, marcenaria e construção naval. (Coelho, 2016).

O desenvolvimento de estudos voltados para a otimização da produção visando atender as exigências e necessidades do mercado consumidor é de extrema importância. Para isso, é necessário quantificar e prognosticar os estoques da floresta, a fim de definir a utilização dos bens advindos da mesma e obter informações para possíveis tomadas de decisão. Dados de diâmetro, altura, área basal e volume das árvores são importantes nas estimativas dos estoques, presente e futuro da produção de madeira de uma floresta (Jesus et al. 2017).

Dentre as variáveis dendrométricas estudadas na floresta, tanto nativa quanto plantada, o volume se constitui em uma das informações com maior importância para o conhecimento do possível potencial florestal de uma região, sendo que o volume individual é o primeiro passo para avaliação da produtividade de povoamentos florestais (Morandi et al, 2017). A cubagem de árvores, além de ser essencial para se caracterizar o comportamento do diâmetro ao longo do tronco, também é eficaz para determinar o volume das árvores abatidas, que pode ser obtido pelo emprego de diferentes fórmulas, das quais se destacam as fórmulas de Smalian, Huber, Newton e Hohenadl.

Para gerar estimativas precisas do volume de madeira de povoamentos florestais são utilizados os modelos de regressão. De posse dos valores volumétricos obtidos por meio de cubagem rigorosa, e da coleta de variáveis dendrométricas como diâmetro à altura do peito (DAP) e altura, por exemplo, faz-se a construção de equações para expressar essa relação (Coelho et. al., 2017).

A utilização de Equações matemáticas de crescimento e produção florestal permite simular a dinâmica natural de um povoamento e prever a sua produção ao longo do tempo, em diferentes possibilidades de exploração (Vanclay, 1994).

Também a quantificação da distribuição diamétrica e suas relações com o sítio, a composição do povoamento, a idade e a densidade são valiosas, tanto para fins econômicos, como para fins biológicos Bailey e Dell (1973). Assim, a distribuição diamétrica surge como um indicador potencial da estrutura da floresta, permitindo distinguir diferentes tipos florestais, além de viabilizar a prognose dos múltiplos produtos (Scolforo, 2006). Jesus et al. (2017) definiram a distribuição diamétrica como sendo um indicador da estrutura do estoque de crescimento, permitindo com certa experiência, a elaboração de conclusões a respeito da estrutura da floresta. 
Desta forma os objetivos deste trabalho foram ajustar equações de volume e de funções probabilísticas para Calophyllum brasiliense, em plantio situado no município de Dueré (TO) bem como estimar sua produtividade.

\section{Material e Métodos}

O estudo foi desenvolvido no ano de 2016, em uma plantação de Calophyllum brasiliense pertencente à empresa JAMP no municipio de Dueré, $221 \mathrm{Km}$ de Palmas (TO). O plantio onde foi realizado o trabalho possui uma área de 35 hectares, e situa-se à uma altitude de 225 metros do nível do mar. O plantio encontra-se no bioma cerrado, onde ocorre o clima estacional com inverno quente e sem qualquer tipo de precipitação durante 5 meses, apresentando fitofisionomia savânica. O clima da região é classificado como sub-úmido a úmido, com deficiência hídrica entre os meses de maio a setembro, e 1500 milímetros de precipitação média ao ano (Köppen e Geiger, 1936).

Os tratos culturais foram feitos desde o primeiro ano sendo: adubação de cobertura, utilizando-se NPK 06-3006 mais micro na dose de $70 \mathrm{~kg} \cdot \mathrm{ha}^{-1}$. O plantio foi conduzido com reforma de aceiros, roçada mecânica de entrelinha, aplicação de herbicida, e combate a formigas. Nos segundo e terceiro anos foi feito manualmente reforma de aceiros, roçada mecânica das entrelinhas, aplicação de herbicida, e combate à formiga. No quarto ano além da manutenção do reflorestamento, ocorreu a desrama de 1/3 da altura total da árvore, após o desbaste sistemático de $25 \%$ das árvores do plantio. Do quinto ano em diante foram feitos apenas manutenção simples tais como reforma de aceiros, combate a formigas, aplicação de herbicidas e inseticidas.

Os dados utilizados nesse trabalho têm como base um inventário florestal contínuo e sistemático, obtido através de parcelas permanentes. O primeiro inventário foi realizado no ano de 2009 quando o plantio apresentava 15 meses sendo medido anualmente até o ano de 2016 aos 94 meses de idade. Onde foram mensuradas as variáveis dendrométricas altura total em metros, DB (diâmetro da base sendo considerado $10 \mathrm{~cm}$ ), DAP (diâmetro a altura do peito com casca $\mathrm{em} \mathrm{cm}$ ) a partir dos 44 meses.

Foram determinadas parcelas primárias, com dimensões 40 × $32 \mathrm{~m}\left(1.280 \mathrm{~m}^{2}\right)$ cada. Nas 24 unidades amostrais foram instaladas 4 subunidades de $8 \times 4 \mathrm{~m} \mathrm{(32}$ $\mathrm{m}^{2}$ ) cada, em uma distribuição sistemática (Figura 1). O tamanho das unidades amostrais primárias e das subunidades foi definido de acordo com o espaçamento do plantio de 4 × $2 \mathrm{~m}$.

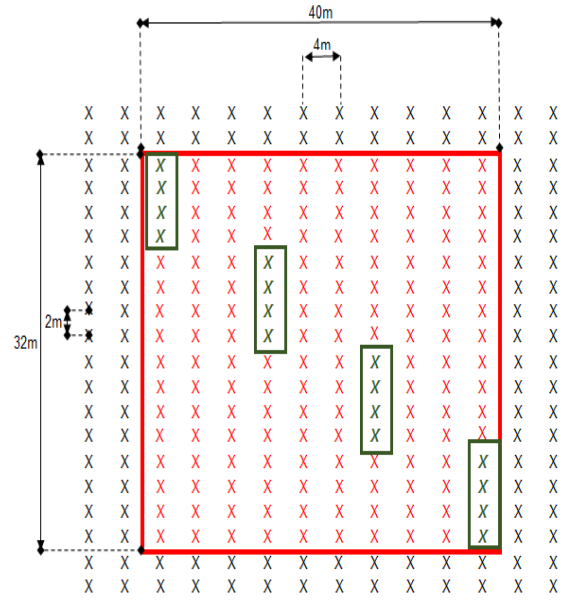

Figura 1: Croqui esquemático sem escala evidenciando a unidade amostral $(40 \times 32 \mathrm{~m})$ e as quatro subunidades amostrais (4 × $2 \mathrm{~m}$ cada) com quatro árvores úteis em cada subunidade.

\section{Ajuste da equação volumétrica}

Foi utilizado o critério da distribuição diamétrica por frequência de cada classe para selecionar as árvores para serem cubadas, sendo que foram selecionadas apenas árvores com DAP superior a 5 centímetros. Para definir quantas árvores deveriam ser cubadas rigorosamente, foi necessário estabelecer uma amostra piloto. A partir desta, o número total de árvores a serem cubadas foi definido através da fórmula de intensidade amostral:

$$
n=\frac{t^{2} *(C V \%)^{2}}{E^{2} \%}
$$

Em que: $\mathrm{t}=$ valor tabelado (Student), $\mathrm{CV} \%=$ coeficiente de variação; $\mathrm{E}=$ erro pré-estabelecido, neste caso utilizado $10 \%, \mathrm{n}=$ número de árvores a serem cubadas rigorosamente.

O volume rigoroso foi determinado a partir da equação 2, da metodologia de Smalian proposta por Machado \& Figueiredo Filho (2006):

$$
v=v_{0}+\sum_{i=1}^{n} v_{i} v_{c}
$$

Em que: $v=$ volume, $v_{0}=$ volume do toco, $v_{i}=$ volume das secções transversais, $v_{c}=$ volume do cone.

Com os dados obtidos na cubagem foram calculados os volumes individuais de cada árvore-amostra. Os dados destas árvores serviram de base para o ajuste das equações volumétricas (Tabela 1), estes foram selecionados a partir da literatura existente sobre o tema (Fernandes et al, 2017).

Tabela 1: Modelos de equações volumétricas para estimativa do volume individual $\mathrm{em}^{3}$ do plantio de Calophyllum brasiliense

\begin{tabular}{|c|c|c|}
\hline $\mathrm{N}^{0}$ & Autor & Funções Volumétricas \\
\hline 1 & $\begin{array}{l}\text { Hohenadl- } \\
\text { Krenn }\end{array}$ & $\mathrm{v}=\beta_{0}+\beta_{1} * \mathrm{~d}+\beta_{2} * \mathrm{~d}^{2}$ \\
\hline 2 & Spurr & $\mathrm{v}=\beta_{0}+\beta_{1} * \mathrm{~d}^{2} * \mathrm{~h}$ \\
\hline 3 & Stoate & $\mathrm{v}=\beta_{0}+\beta_{1} * \mathrm{~d}^{2} *\left(\mathrm{~d}^{2} * \mathrm{~h}\right)+\beta_{2} * \mathrm{~h}$ \\
\hline 4 & Näslund & $\begin{array}{c}\mathrm{v}=\beta_{1} * \mathrm{~d}^{2}+\beta_{2} *\left(\mathrm{~d}^{2} * \mathrm{~h}\right)+\beta_{3} *\left(\mathrm{~d} * \mathrm{~h}^{2}\right) \\
+\beta_{4} * \mathrm{~h}\end{array}$ \\
\hline 5 & Meyer & $\begin{array}{r}\mathrm{v}=\beta_{0}+\beta_{1} * \mathrm{~d}+\beta_{2} * \mathrm{~d}^{2} * \beta_{3} *(\mathrm{~d} * \mathrm{~h}) \\
+\beta_{4} *\left(\mathrm{~d}^{2} * \mathrm{~h}\right)\end{array}$ \\
\hline 6 & $\begin{array}{l}\text { Meyer } \\
\text { (modificado) }\end{array}$ & $\begin{aligned} \mathrm{v}=\beta_{0}+\beta_{1} * \mathrm{~d}+\beta_{2} * & \mathrm{~d}^{2}+\beta_{3} *(\mathrm{~d} * \mathrm{~h}) \\
& +\beta_{4} *\left(\mathrm{~d}^{2} * \mathrm{~h}\right) \\
& +\beta_{5} * \mathrm{~h}\end{aligned}$ \\
\hline 7 & Spurr & $\operatorname{lnv}=\beta_{0}+\beta_{1} * \ln \left(\mathrm{d}^{2} \mathrm{~h}\right)$ \\
\hline 8 & $\begin{array}{l}\text { Schumacher- } \\
\text { Hall }\end{array}$ & $\operatorname{lnv}=\beta_{0}+\beta_{1} * \ln d+\beta_{2} * \operatorname{lnh}$ \\
\hline
\end{tabular}
em Dueré - TO.

Em que: $\mathrm{v}=$ volume individual $\mathrm{em} \mathrm{m}^{3}, \mathrm{~h}=$ altura total $(\mathrm{m}) \mathrm{e} \mathrm{d}=$ diâmetro a altura do peito em $\mathrm{cm} ; \beta_{0}, \beta_{1}, \beta_{2}, \beta_{3}, \beta_{4}$ e $\beta_{5}=$ coeficientes da regressão.

\section{Função de densidade e probabilidade (fdp)}

Para testar as funções de densidade e probabilidade, foram mensuradas 257 árvores medidas no inventário florestal contínuo desde os 15 meses de idade. Foram medidos o DAP de todas as árvores acima de $5 \mathrm{~cm}$ das parcelas, além das correspondentes alturas totais em metros. A partir destes dados, os diâmetros foram distribuídos em intervalos de classes de $2 \mathrm{~cm}$, com a finalidade de realizar o ajuste das distribuições.

As $f d p$ 's foram ajustadas no software FitFd disponível gratuitamente pelo projeto NeuroForest, onde foram selecionadas as seis (num total de 150) equações que melhor representaram o ajuste dos dados. Os parâmetros 
das funções de densidade de probabilidade analisadas foram estimados pelo método da máxima verossimilhança.

O teste de Kolmogorov-Smirnov foi utilizado a um nível de 5\% de probabilidade de confiança para realizar a verificação da aderência das funções aos dados estudados. $\mathrm{O}$ mesmo foi usado para testar as hipóteses de $\mathrm{H}_{0}$ e $\mathrm{H}_{1}$ do teste bilateral. Sendo que $\mathrm{H}_{0}=$ os diâmetros observados seguem as distribuições propostas e $\mathrm{H}_{1}=$ os diâmetros observados não seguem as distribuições propostas, sendo determinado pela equação 3 :

$$
\text { Dcalc }=\frac{\sup _{*}\left(F o_{x}-F e_{x}\right)}{n}
$$

Em que: $F e=$ frequência acumulada observada; $F o=$ frequência estimada pela função densidade de probabilidade; $\mathrm{n}=$ número de observações.

Para a seleção da função de densidade probabilística de melhor aderência foram utilizadas as estatísticas de ajuste e precisão, coeficiente de determinação ajustado $\left(\mathrm{R}^{2} \mathrm{aj}\right)$ e erro padrão da estimativa em porcentagem (Syx\%) calculadas com os valores observados e estimados pelas $f d p$ 's do número de árvores por classe diamétrica. Tais estatísticas de ajuste e precisão são amplamente conhecidas no meio florestal e sua descrição detalhada pode ser encontrada em Maraschin et al. (2017). Por último realizou-se a análise gráficas da distribuição dos resíduos das equações.

\section{Cálculo do Incremento Periódico (IP) e Incremento Médio Anual (IMA)}

Estes foram determinados pelas equações descritas por Schneider (1993), sendo:

$$
\begin{array}{cc}
I P=X_{n+1}-X_{n} & (\text { Equação } 4) \\
\text { e } \quad I M A=\frac{X_{I}}{I} & (\text { Equação 5) }
\end{array}
$$

Em que: $X_{n}=$ variável dendrométrica no início do período; $\mathrm{X}_{\mathrm{n}+1}=$ variável dendrométrica no final do período, $\mathrm{VI}=$ variável na idade I; I = idade do povoamento.

\section{Resultados}

Na determinação da estatística descritiva (Tabela 2), observa-se que os diâmetros com casca, as alturas totais e o volume variam de $3,50 \mathrm{~cm}$ a $12,10 \mathrm{~cm} ; 2,87 \mathrm{~m}$ a $8,20 \mathrm{~m}$; 0,0043 a $0,067 \mathrm{~m}^{3}$, respectivamente. Existe uma baixa amplitude das variáveis dendrométricas bem como baixo coeficiente de variação (valores $\leq 20 \%$ para todas variáveis). Resultados assim são encontrados em povoamentos homogêneos explicados pelo regime de manejo e tratos silviculturais.

Para a variável IMA, Ciriello (2010) encontrou valores superiores aos encontrados neste trabalho para DAP e Ht, sendo um incremento médio em altura de $1,4 \mathrm{~m} / \mathrm{ano}$ e um incremento médio em diâmetro da ordem de $1,82 \mathrm{~cm} / \mathrm{ano}$ aos 7 anos de idade para a espécie Calophyllum brasiliense.

Tabela 2: Estatística descritiva das variáveis dendrométricas DAP $(\mathrm{cm})$, Ht $(\mathrm{m})$ e $\mathrm{Vol}\left(\mathrm{m}^{3}\right)$ do plantio comercial de Calophyllum brasiliense em Dueré (TO) aos 94 meses de idade

\begin{tabular}{lccc}
\hline Estatísticas & $\mathrm{DAP}(\mathrm{cm})$ & $\mathrm{Ht}(\mathrm{m})$ & $\mathrm{Vol}\left(\mathrm{m}^{3}\right)$ \\
\hline Média & 7,477 & 5,434 & 0,0274 \\
Erro padrão & 0,102 & 0,048 & 0,0240 \\
Mediana & 7,448 & 5,500 & 0,0268 \\
Moda & 7,321 & 5,000 & 0,0340 \\
Desvio padrão & 1,711 & 0,808 & 0,0109 \\
Variância da amostra & 2,928 & 0,653 & 0,0001 \\
CV\% & 17,7 & 19,3 & 8,3100 \\
Intervalo & 8,6 & 5,33 & 0,0634
\end{tabular}

\begin{tabular}{lccc} 
Mínimo & 3,50 & 2,87 & 0,0043 \\
Máximo & 12,1 & 8,20 & 0,0678 \\
IMA & 1,27 & 0,88 & 0,00352 \\
Contagem & 257 & 257 & 257 \\
\hline
\end{tabular}

Em que: DAP $=$ Diâmetro a 1,3 metros de altura, $\mathrm{Ht}=$ altura total medida em metros, $\operatorname{Vol}\left(\mathrm{m}^{3}\right)=$ volume individual total com casca.

A estrutura diamétrica utilizada para o ajuste das $f d p$ 's demonstrou que a comunidade arbórea é composta em sua maioria por árvores finas, sendo $53,1 \%$ abaixo de $10 \mathrm{~cm}$ de DAP e baixas, sendo $46,5 \%$ menores que $7 \mathrm{~m}$ de altura total que comprova que essa comunidade está em estágio inicial de crescimento (tabela 3 ).

Tabela 3: Frequência absoluta, frequência relativa e frequência absoluta estimada por classe diamétrica para plantio comercial de Calophyllum brasiliense aos .94 meses no município de Dueré (TO)

\begin{tabular}{ccccc}
\hline Centro de classe & FA & FR\% & FAE & $\mathrm{N}^{\mathbf{0}}$ de árvores cubadas \\
\hline 5 & 1 & 0,389 & 0 & 1 \\
7 & 32 & 12,45 & 29 & 16 \\
9 & 104 & 40,47 & 107 & 11 \\
11 & 89 & 34,63 & 89 & 5 \\
13 & 30 & 11,67 & 30 & 12 \\
15 & 1 & 0.389 & 2 & 1 \\
\hline Total & 257 & 100 & 257 & 46 \\
\hline
\end{tabular}

Em que: $\mathrm{FA}=$ frequência absoluta; $\mathrm{FR} \%$ = frequência relativa em percentagem, e FAE = Frequência absoluta estimada

Verifica-se na tabela 4 os ajustes entre as equações ajustadas, identificando baixa variabilidade entre elas pois os coeficientes de determinação ajustado ( $\left.R^{2} a j\right)$ variam entre 0,92 e 0,98 , e todos os coeficientes de variação ficaram abaixo dos $15 \%$ (indicando baixa dispersão dos dados). Para o erro padrão da estimativa (Syx\%) a amplitude foi de 12,21\% sendo considerada aceitável indicando o quando os valores estimados diferem em média dos observados.

Tabela 4: Estimativas dos parâmetros estatísticos das equações

\begin{tabular}{|c|c|c|c|c|c|c|c|c|c|}
\hline Função & $\beta_{0}$ & $\beta_{1}$ & $\beta_{2}$ & $\beta_{3}$ & $\beta_{4}$ & $\beta_{5}$ & $\begin{array}{l}R^{2} \\
\text { aj }\end{array}$ & $\begin{array}{c}\text { Syx } \\
\%\end{array}$ & $\begin{array}{c}\mathrm{CV} \\
\%\end{array}$ \\
\hline Hohenad & 0,0 & 0,00 & 0,00 & - & . & - & 0,9 & 14, & $\begin{array}{c}14, \\
9\end{array}$ \\
\hline 1 Krenn & & 3,10 & & & & & & $\begin{array}{l}45 \\
9,9\end{array}$ & \\
\hline Spurr & $\begin{array}{l}0,0 \\
05\end{array}$ & $\begin{array}{l}\text { E- } \\
05\end{array}$ & - & - & - & - & $\begin{array}{c}0,9 \\
6\end{array}$ & 9 & $\begin{array}{c}10, \\
3\end{array}$ \\
\hline Stoate & $\begin{array}{l}0,0 \\
09\end{array}$ & $\underset{0,6}{2,3^{\mathrm{E}-}}$ & $\begin{array}{c}2,4^{\mathrm{E}-} \\
0,5\end{array}$ & $\begin{array}{c}0,00 \\
2\end{array}$ & - & - & $\begin{array}{c}0,9 \\
7\end{array}$ & $\begin{array}{c}8,2 \\
2\end{array}$ & 8,4 \\
\hline Näslund & $\begin{array}{l}0,0 \\
03\end{array}$ & $\begin{array}{c}8,3^{\mathrm{E}-} \\
0,5\end{array}$ & $\begin{array}{c}1,3^{\mathrm{E}-} \\
0,5\end{array}$ & $\begin{array}{c}-1^{\mathrm{E}-} \\
0,6\end{array}$ & $\begin{array}{c}0,00 \\
02\end{array}$ & - & $\begin{array}{c}0,9 \\
7\end{array}$ & $\begin{array}{c}8,0 \\
3\end{array}$ & 8,2 \\
\hline Meyer & $\begin{array}{c}0,0 \\
16\end{array}$ & $\begin{array}{c}- \\
0,00 \\
6\end{array}$ & $\begin{array}{c}0,00 \\
03\end{array}$ & $\begin{array}{c}0,00 \\
07\end{array}$ & $\begin{array}{c}-2^{\mathrm{E}-} \\
0,5\end{array}$ & - & $\begin{array}{c}0,9 \\
7\end{array}$ & $\begin{array}{c}8,2 \\
7\end{array}$ & 8,5 \\
\hline $\begin{array}{l}\text { Meyer } \\
\text { Mod. }\end{array}$ & $\begin{array}{c}0,0 \\
11\end{array}$ & $\begin{array}{c}- \\
0,00 \\
5\end{array}$ & $\begin{array}{c}0,00 \\
03\end{array}$ & $\begin{array}{c}0,00 \\
05\end{array}$ & $\begin{array}{c}-9,{ }_{0,6}^{\text {E- }} \\
\text { - }\end{array}$ & $\begin{array}{c}0,00 \\
08\end{array}$ & $\begin{array}{c}0,9 \\
7\end{array}$ & $\begin{array}{c}8,3 \\
8\end{array}$ & 8,6 \\
\hline $\begin{array}{l}\text { Spurr } \\
\text { log. }\end{array}$ & $\begin{array}{c}- \\
8,9 \\
17\end{array}$ & $\begin{array}{c}0,81 \\
3\end{array}$ & - & - & - & - & $\begin{array}{c}0,9 \\
7\end{array}$ & $\begin{array}{c}2,5 \\
1\end{array}$ & 7,9 \\
\hline $\begin{array}{l}\text { Schumac } \\
\text { her-Hall }\end{array}$ & $\begin{array}{c}- \\
9,0 \\
51\end{array}$ & $\begin{array}{c}1,29 \\
2\end{array}$ & $\begin{array}{c}1,26 \\
6\end{array}$ & - & - & - & $\begin{array}{c}0,9 \\
8\end{array}$ & $\begin{array}{c}2,2 \\
4\end{array}$ & 7,5 \\
\hline
\end{tabular}
matemáticas testadas para descrever o volume individual de Calophyllum brasiliense. em Dueré-TO aos 94 meses de idade

Em que: $\beta_{0}, \beta_{1}, \beta_{2}, \beta_{3}, \beta_{4}$ e $\beta_{5}=$ parâmetros das equações; $\mathrm{R}^{2} \mathrm{aj}=$ coeficiente de determinação ajustado; $\mathrm{CV} \%=$ coeficiente de variação em percentagem; $\mathrm{F}=$ valor de $\mathrm{F}$; *= significância a 5\%; ns= não significativo.

A análise gráfica de dispersão de resíduos (Figura 1), entre os valores observados e estimados, mostrou que a função matemática descrita por Schumacher-Hall: $\ln (\mathrm{v})=$ $\beta_{0}+\beta_{1}+\operatorname{lnd} * \beta_{2} * \operatorname{lnh}$ apresentou ajuste mais adequado para o conjunto de dados entre as equações testadas 


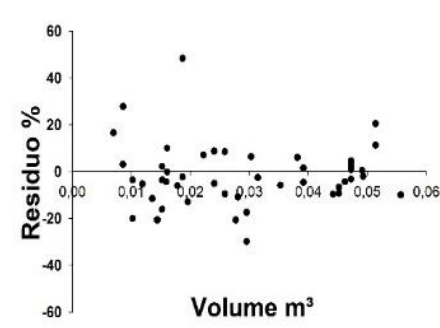

Hohenadl-Krenn
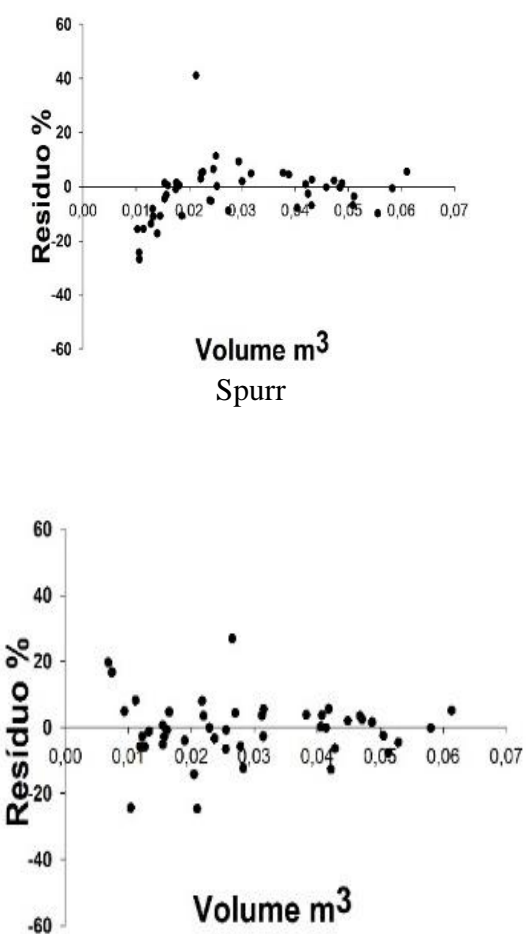

Meyer
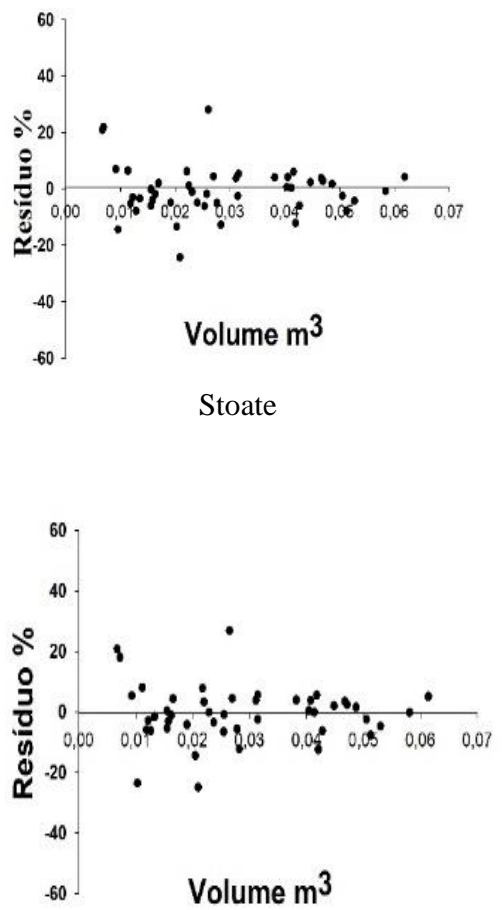

Meyer modificado
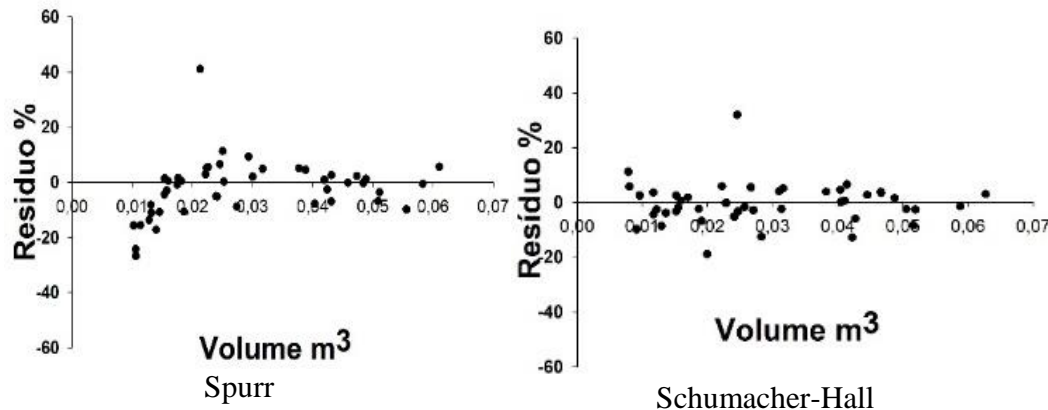

Schumacher-Hall

Figura 1: Distribuição percentual dos resíduos das equações testadas para determinação do volume individual.

Esta função volumétrica é muito usada para estimar o volume individual de plantios comerciais de espécies florestais, principalmente do gênero Eucalyptus e Pinus. A análise gráfica dos resíduos mostrou que a função de Schumacher-Hall foi superior, pois não apresentou tendenciosidade para a estimativa do volume total com $\mathrm{R}^{2}$ aj de 0,98 e Syx $\%=7,5$. Por ser a função que melhor descreve o conjunto de dados, a hipótese de não existir diferenças significativas entre o conjunto de dados, foi testada somente para esta. (Figura 2).

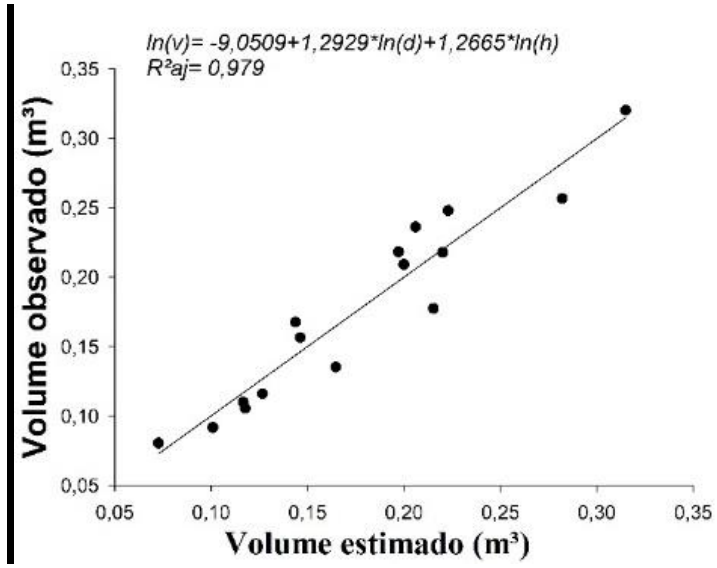

Figura 2: Reta da equação de Schumacher-Hall com os dados estimados para do plantio comercial de Calophyllum brasiliense aos 94 meses de idade em Dueré-TO.

Foi realizado o teste de Kolmogorov-Smirnov para comprovar a normalidade dos resíduos, o que pôde ser concluído considerando $\alpha=0,05$ e $\mathrm{n}=46$. Obteve-se o valor de $D_{n} 0,21<0,40$, o que se conclui que não existe 
diferença significativa entre os valores reais e os estimados considerando um nível de significância de 5\%, sendo assim, confirmou-se que a função matemática de Schumacher-Hall pode ser utilizado para o cálculo do volume do plantio.

Na determinação das funções probabilísticas, a função matemática que melhor descreve os dados observados foi a função $Y=e^{\beta 0+\left(\frac{-\beta 1}{X}\right)+\beta 3 * \ln (X)} \quad$ (função 2) por apresentar os melhores desempenhos, com valor muito alto de $\mathrm{R}^{2}$ aj e valor baixo para Syx \%. Esta função pode ser classificada como do tipo exponencial e pode ser

\begin{tabular}{|c|c|c|c|c|c|c|c|c|}
\hline$f d p$ 's & Funções & $\beta_{0}$ & $\beta_{1}$ & $\beta_{2}$ & $\beta_{3}$ & $R^{2}$ aj & Syx $\%$ & $\mathrm{CV}$ \\
\hline 1 & $\mathrm{Y}=\beta_{0} * \operatorname{sen}\left(\beta_{1} * \mathrm{x}+\beta_{2}\right)+\beta_{3}$ & 51,31 & 2,550 & 23,99 & 47,8 & 0,98 & 1,77 & 8,78 \\
\hline 2 & $Y=\exp ^{\beta_{0}+\left(\frac{-\beta_{1}}{X}\right)+\beta_{3} * \ln (X)}$ & 1,128 & $-3,124$ & 0,649 & - & 0,99 & 1,66 & 8,76 \\
\hline 3 & $\left.\mathrm{Y}=\beta_{0} * \beta_{1} * X^{\beta 2}\right)$ & $1,45^{\mathrm{E}-04}$ & 0,315 & 10,75 & - & 0,96 & 1,67 & 8,77 \\
\hline 4 & $\mathrm{Y}=\mathrm{x} /\left(\beta_{0}+\beta 1 * \mathrm{x}+\beta_{2} * \sqrt{X}\right)$ & 9,35 & 1,008 & 6,119 & - & 0,96 & 1,67 & 8,77 \\
\hline 5 & $Y=\frac{\beta_{0}}{\beta_{1}} * \mathrm{x}^{\beta 0-1} * \exp ^{-\mathrm{x}^{\frac{\beta_{0}}{\beta 1}}}$ & $7,46^{\mathrm{E}+08}$ & $3,07^{\mathrm{E}+09}$ & $\begin{array}{l}3,07^{\mathrm{E}+} \\
09\end{array}$ & - & 0,87 & 2,17 & 20,7 \\
\hline 6 & $Y=\frac{\beta_{0}}{\beta_{1}} * \frac{\mathrm{x}^{\beta 0-1}}{\beta_{1}} * \exp ^{-1 \beta_{3}} * \frac{\mathrm{x}^{\beta_{0}}}{\beta_{1}}$ & 12,81 & $6,02^{\mathrm{E}+12}$ & - & - & 0,80 & 0,17 & 19,5 \\
\hline
\end{tabular}

Em que: $\beta_{0}, \beta_{1}, \beta_{2} e \beta_{3}=$ coeficientes da regressão, $\mathrm{R}^{2} \mathrm{aj}=$ Coeficiente de determinação ajustado, Syx\%= Erro padrão da estimativa em porcentagem $\mathrm{CV}=$ Coeficiente de variação em porcentagem.

A análise gráfica dos resíduos das funções de densidade e probabilidade com maior correlação entre os

fdp 1

fdp 2

valores observados e estimados, indicam que a $f d p 2$ foi a que apresentou melhor aderência dos dados (Figura 3). comparado ao modelo de Weibull, a qual tem sido utilizada na maioria dos estudos, por ser uma função flexível e pelo fato de seus parâmetros serem facilmente correlacionados com características dos povoamentos, pela simplicidade de ajuste (Campos \& Leite, 2013) e pelo fato de a função já ser exaustivamente estudada na área florestal.

Tabela 5: Parâmetros estimados das funções de distribuição diamétrica para plantio comercial de Calophyllum brasilienseaos 94 meses

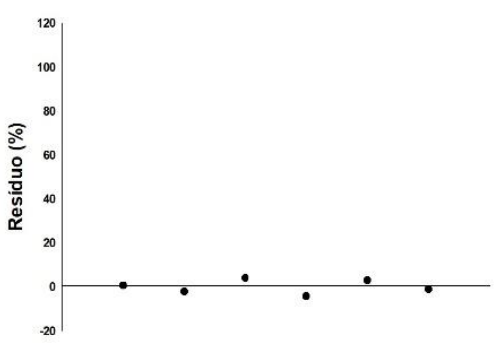

fdp 4
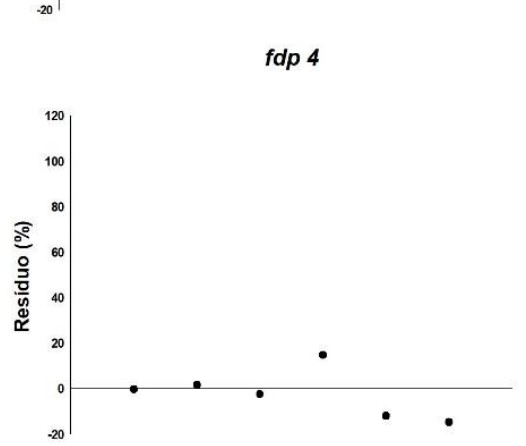

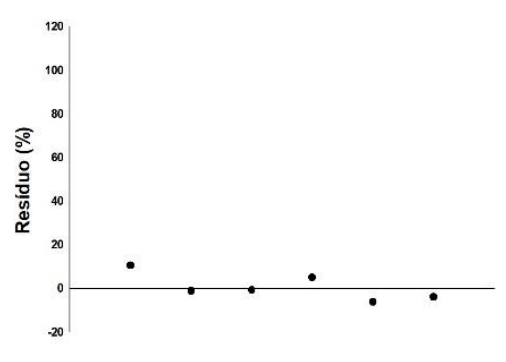

fdp 5

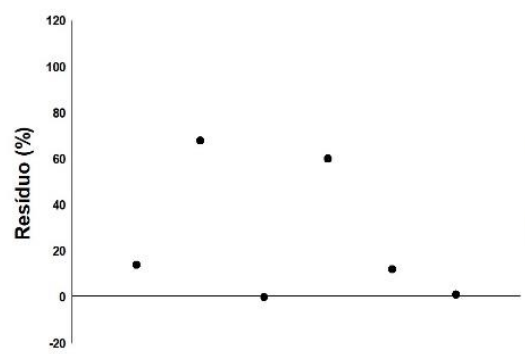

fdp 3

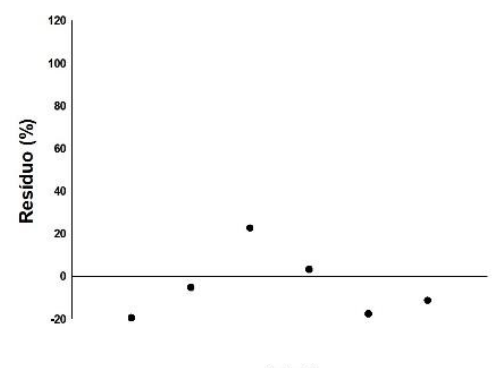

$f d p 6$

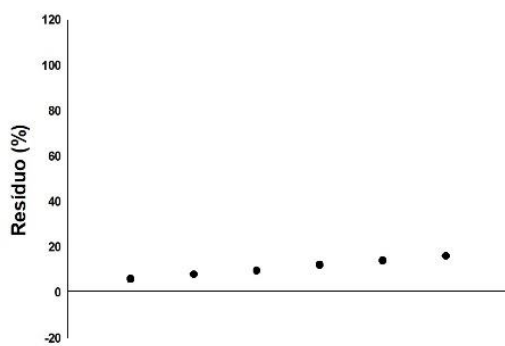

Figura 3: Distribuição percentual dos resíduos das funções de densidade e probabilidade testadas para o plantio comercial de Calophyllum brasiliense em Dueré (TO) aos 94 meses.

O teste de Kolmogorov-Smirno $/ \mathrm{v}$ (K-S) foi utilizado para testar as hipóteses de $\mathrm{H}_{0}$ e $\mathrm{H}_{1}$ do teste bilateral para as funções probabilísticas, onde, $\mathrm{H}_{0}=$ os diâmetros observados seguem distribuição normal e $\mathrm{H}_{1}=$ os diâmetros observados não seguem distribuição normal. $\mathrm{O}$ valor encontrado de $\mathrm{D}_{\text {calc }}=0,0156$ (função 2) é menor que o valor de $\mathrm{D}_{\text {tab }}(95 \%)=0,120$ não rejeitamos a hipótese nula e concluímos que não existem evidência de que as variâncias obtidas para o conjunto de dados, são diferentes, ao nível de significância de 5\%. Já para os $\mathrm{D}_{\text {calc }}=0,1984$ (funções 1,3 e 4) rejeitamos a hipótese Ho inferindo que existe diferença entre os valores observados e os valores estimados.

Observou-se que $68,48 \%$ dos indivíduos estimados pela $f d p 2$ encontram-se entre no intervalo entre a média quadrática $(9,97 \mathrm{~cm}) \pm 0$ desvio Padrão $(1,67 \mathrm{~cm})$ indicando que os dados seguem uma distribuição normal de frequência que é característico de florestas plantadas. 


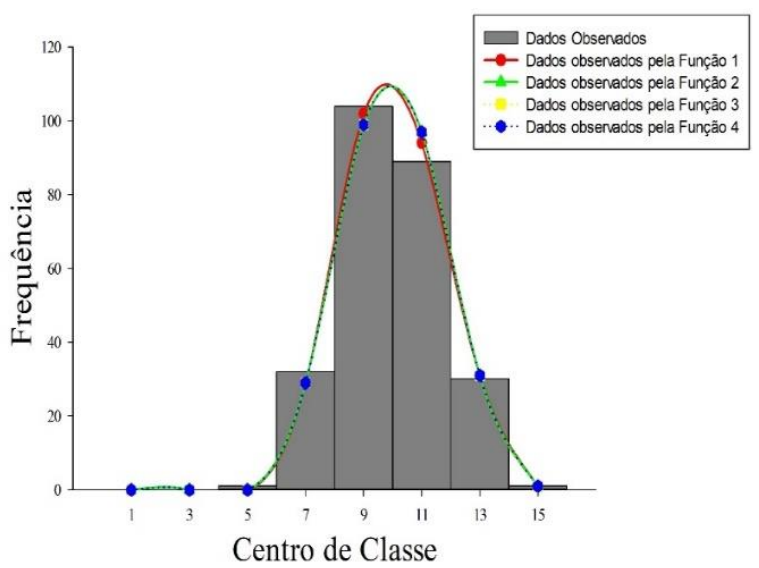

Figura 4: Frequência observada e estimada pelas fdp's para Calophyllum brasiliense em Dueré (TO).

O teste qui-quadrado $(\chi 2)$ foi utilizado para verificar se as frequências observadas não são diferentes das frequências estimadas a um nível de $5 \%$ de probabilidade de confiança. Para a $f d p$ selecionada o valor $\chi 2$ calculado $(0.028)$ foi $<\chi 2$ tabelado $(0,71)$ não detectando diferença significativa entre os valores observados e os estimados pela $f d p 2$.

O incremento médio anual para esse povoamento, foi de $11,78 \mathrm{~m}^{3} \mathrm{ha}^{-1}$ (figura 5) aos 94 meses de idade de idade, e seu incremento periódico foi de $40,38 \mathrm{~m}^{3} \mathrm{ha}^{-1}$ para um volume total estimado de $92,3 \mathrm{~m}^{3} \mathrm{ha}^{-1}$ aos mesmos 94 meses de idade. Valor superior ao estudo feito por Kallil Filho et al, (2012) que em seus resultados de seleção maçal de Calophyllum brasiliense em plantios comerciais com espaçamento de $3 \times 2 \mathrm{~m}$, obtiveram valores de $3,33 \mathrm{~m}^{3} \cdot \mathrm{ha}^{-1}$ aos 60 meses de idade.

\section{Incremento ao longo do tempo}

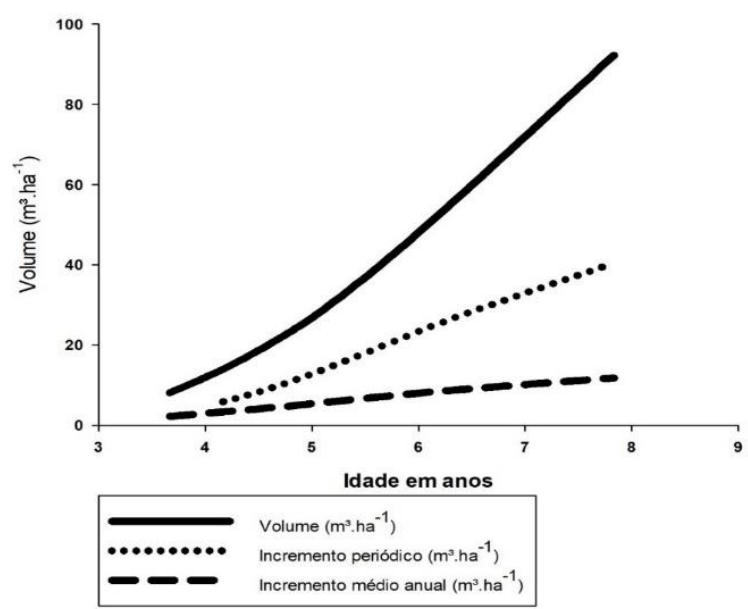

Figura 5: Incrementos periódico, médio, e volume total de Calophyllum brasilienses em Dueré (TO).

\section{Discussão}

A espécie Calophyllum brasiliense se mostrou uma ótima alternativa para o mercado madeireiro brasileiro, visto que apresentou um crescimento satisfatório nas condições de plantio comercial.

O comportamento de crescimento horizontal de Calophyllum brasiliense é semelhante as espécies tradicionalmente plantadas dos gêneros Eucalyptus e Pinus pois ficou caracterizado distribuição normal aos 94 meses de idade indicando boa adaptação da espécie em plantios comerciais.
A equação matemática selecionada para determinação volumétrica, descrita por Schumacher-Hall é comumente utilizada para estimar o volume de espécies dos gêneros Eucalyptus e Pinus. Esse pode ser comprovado pelo trabalho de Rocha et al. (2010) ao avaliarem métodos de estimativas de volume para povoamento de Eucalyptus urophylla com 57 meses de idade no planalto da conquista (BA) determinaram que a partir do inventário tradicional, o modelo volumétrico que apresentou o melhor desempenho foi o de Schumacher-Hall (logarítmica).

Também Lopes (2014) ao determinar o comprimento ótimo das seções na cubagem rigorosa de árvores de Eucalyptus híbrido clonal (GG100) aos cinco anos de idade para ajuste de modelos volumétricos.

A função exponencial descrita por: $Y=\exp ^{\beta_{0}+\left(\frac{-\beta_{1}}{X}\right)+\beta_{3} * \ln (X)}$ foi selecionada para descrever a distribuição diamétrica, por apresentar inexistência de tendenciosidade nas estimativas, do plantio comercial é amplamente empregada no setor florestal (Machado \& Figueiredo Filho, 2009).

Os valores de produtividade para as variáveis DAP e altura total em metros corroboram com valores encontrados para a espécie aos 7 anos de idade em plantios comerciais. Volpato et al. (1972) encontraram valores de 7,8 metros de média para altura total e de $10 \mathrm{~cm}$ de DAP médio em Manaus para plantio com espaçamento de $3 \mathrm{x}$ $2 \mathrm{~m}$. Em Paranaguá (PR) em plantio com a mesma idade e espaçamento de $3,0 \mathrm{~m} \times 1,5 \mathrm{~m}$, Carvalho et al (2003) encontraram valores médios de $4,68 \mathrm{~m}$ para altura total e de $3,7 \mathrm{~cm}$ de DAP.

Piotto (2005) avaliando plantio de Calophyllum brasiliense, observou no crescimento inicial ( 7 anos) incremento médio anual em altura de $1,4 \mathrm{~m}$ e incremento médio em diâmetro de 1,82 cm. Petit e Montagnini (2006) encontraram para altura total valor médio de $1,5 \mathrm{~m}$ e para DAP 1,6 cm. Kallil Filho et al. (2012) encontraram para altura total $5,17 \mathrm{~m}$ e para DAP $27 \mathrm{~cm}$ aos 6 anos de idade em Mococa (SP) em plantios comerciais da espécie e Niere et al (2018) encontraram valores médios de altura total igual a $1,77 \mathrm{~m}$ e $1,40 \mathrm{~cm}$ de DAP aos 52 meses de idade no sul de Minas Gerais para plantios em sistemas agroflorestais.

Para variável volume, o valor encontrado está dentro de valores observados em plantios para a espécie. Em Manaus (AM) Alencar et al (1981) encontraram um volume médio de $1,44 \mathrm{~m}^{3} \cdot \mathrm{ha}^{-1}$ ano em plantio sob sombra de floresta primária aos 7 anos de idade. Kallil Filho et al. (2012) encontraram volume médio de $43,4 \mathrm{~m}^{3} \cdot \mathrm{ha}^{-1}$ aos 6 anos de idade. Na Costa Rica para volume aos 11 aos de idade foram encontrados valores de IMA $14,4 \mathrm{~m}^{3} \cdot \mathrm{ha}^{-1}$ (Petit e Montagnini, 2006). Nieri et al (2018) encontraram para plantios em sistemas agroflorestais com a espécie um volume médio de $1,4 \mathrm{~m}^{3}$ aos 52 meses de idade no sul de Minas Gerais.

\section{Conclusões}

A comunidade vegetal é composta principalmente por árvores finas, baixas e com baixa taxa de mortalidade aos 94 meses.

A equação volumétrica de Schumacher-Hall pode ser usada em plantios comerciais de Calophyllum brasiliense. A função probabilística descrita por: $Y=$ $e^{1,128+\left(-\frac{3,124}{X}\right)+0,649 * \ln (X)}$ pode ser usada para a modelagem da distribuição diamétrica da espécie em plantios comerciais. 
O plantio obteve um crescimento considerado satisfatório quando comparado com outros plantios da mesma espécie e outras culturas pelo Brasil, o que torna atrativa a comercialização da sua madeira, além de uma opção às culturas já usadas no país.

\section{REFERÊNCIAS BIBLIOGRÁFICAS}

Alencar, JC; Fernandes, NP; Loureiro, AA.(1981) Desenvolvimento de árvores nativas em ensaios de espécies 2. Jacareúba (Calophyllum angulare A.C. Smith). Acta Amazônica.[online]. vol.11, $\mathrm{n}^{\mathrm{o}}$ 2, p.357-370. Doi: 10.1590/1809-43921981112357.

Busato, Jader Galba et al. Efeito do extrato húmico solúvel em água e biofertilizante sobre o desenvolvimento de mudas de Callophyllum brasiliense. Pesquisa Florestal Brasileira , v. 36, n. 86, p. 161-168, jun. 2016 doi: 10.4336/2016.pfb.36.86.1024

Campos, J. C. C., Leite, H. G. Mensuração Florestal: perguntas e respostas. 4. ed. Viçosa: Editora UFV, 2013. $605 \mathrm{p}$

Carvalho, P. E. R. Espécies arbóreas brasileiras. v. 1. Brasília: Embrapa Informação Tecnológica; Colombo/PR: Embrapa Florestas, 2003. 1039 p

Ciriello, V. (2010) Crescimento inicial e nutrição de Guanandi (Calophyllum brasiliense Cambess) em função de N, P, K e saturação por bases do solo. Dissertação (Mestrado em Ciência Florestal) - UNESP. Botucatu, 108 p.

Coelho, M.C.B, (2016). EPIDOMETRIA DE Calophyllum brasiliense Cambess. EM PLANTIOS COMERCIAIS, Brasil. Tese (Doutorado em engenharia florestal), Publicação PPG EFL. TD 061/2016, Departamento de Engenharia Florestal, Universidade de Brasília, Brasília, DF,100p.

Coelho, M.C.B; et al (2017) . Funções de densidade de probabilidade para a estimativa da distribuiçao diamétrica de plantios de Calophyllum brasiliense Cambess. Revista Forestal Mesoamericana Kurú , Cartago, Costa Rica, v. 14, n. 34, p. 45-52, Doi:10.18845/rfmk.v14i34.3002

Fernandes, A.M.V; et al. Equações volumétricas para Carapa guianensis Aubl. e Swietenia macrophylla King em sistema silvipastoril na Amazônia. Nativa, Sinop, MT, v. 5, n. 1, p. 73-77, fev. 2017. Doi:10.5935/23187670.v05n01a12.

Ferreira,J., C., S.(2011) Análise da estrutura diamétrica em povoamentos de florestas plantadas a partir de funções de densidade de probabilidade. Dissertação. Mestrado em ciências florestais. Brasília. DF.116 p.

Jesus, C., M.; Miguel, E., P.; Rezende, A., V.; GASPAR, R., de O.; GATTO, A.; Valadão, M., B., X.; Carrijo, J., V. (2017), Funções de densidade de probabilidade para estimativa das distribuições de variáveis dendrométricas em um povoamento clonal de eucalipto. Revista Espacios. Vol. 38, no 16. 30p.

Kallil Filho, A.N. Wedling, I.; Ribeiro, R. M. de. Seleção de Guanandi em plantios comerciais. Comunicado Técnico 299.Colombo, dez. 2012.

Köppen, Graz. Handbuch der Klimatologie. In: KÖPPEN, Graz; GEIGER, München. Handbuch der Klimatologie. 35. ed. Berlin: [s.n.], 1936. p. 1-44. v. 17. Disponível em: <https://upload.wikimedia.org/wikipedia/commons/3/ 3c/Das_geographische_System_der_Klimate_\%281936\% 29.pdf>.

Lopes, PF. (2014). Comprimento ótimo das seções na cubagem rigorosa de árvores de Eucalipto para ajustes de modelos volumétricos e de taper. Dissertação, Universidade de Brasilia (UNB), Brasil, 29p.

Machado SA, Figueiredo-Filho A. Dendrometria. 2. ed. Guarapuava: Unicentro; 2006.

MACHADO, Sebastião do Amaral et al (2009). Funções de distribuição diamétrica em um fragmento de Floresta Ombrófila Mista. Cienc. Rural [online]. vol.39, n.8, pp.2428-2434. Doi:10.1590/S0103-84782009000800024.

MARANGON, Gabriel Paes et al . MODELAGEM DA DISTRIBUIÇÃ̃ DIAMÉTRICA DE ESPÉCIES LENHOSAS DA CAATINGA, SEMIÁRIDO PERNAMBUCANO. Ciênc. Florest., Santa Maria , v. 26, n. 3, p. 863-874, Sept. 2016 . Doi: $10.5902 / 1980509824214$.

Maraschin, D.; Finger, A.; Loreto, A. Definição intervalar e análise de qualidade da função densidade de probabilidade da variável aleatória contínua com distribuição Beta. Scientia Plena, Aracajú, v. 13, n. 4, p. 1-10, maio. 2017. Doi: doi.org/10.14808/sci.plena.2017.049904.

Morandi, P.S. et al. Influência da disponibilidade de luz na qualidade de mudas e nutrição mineral de Calophyllum brasiliense Cambess. (Calophyllaceae). Revista árvore , Viçosa, v. 41, n. 2, p. 1-10, abr. 2017. Disponível em: <http://dx.doi.org/10.1590/1806-90882017000200016 >. Doi: 10.1590/1806-90882017000200016.

NIERI, Erick Martins et al.(2018) COMPORTAMENTO SILVICULTURAL DE ESPÉCIES FLORESTAIS EM ARRANJO PARA INTEGRAÇÃO PECUÁRIA FLORESTA. FLORESTA, [S.1.], v. 48, n. 2, p. 195-202 doi:http://dx.doi.org/10.5380/rf.v48i2.54744.

PETIT, B.; MONTAGNINI, F.(2006) Growth in pure and mixed plantations of tree species used in reforesting rural areas of the humid region of Costa Rica, Central América. Forest Ecology and Management, n. 233, p. 338-343.

Piotto, D;(2005). Projeto técnico de reflorestamento fazenda São Gabriel. 27p.

Robert L. Bailey, T. R. Dell; Quantifying Diameter Distributions with the Weibull Function, Forest Science, Volume 19, Issue 2, 1 June 1973, Pages 97-104, https://doi.org/10.1093/forestscience/19.2.97

Rocha, TB. Cabacinha, CD. Almeida, RC; Paula, A.; Santos, RC. Avaliação de métodos de estimativa de volume para um povoamento de Eucalyptus urophylla $\mathrm{S}$. T. Blake no Planalto da Conquista- BA. Enciclopédia Biosfera, Centro Científico Conhecer - Goiânia, v.6, N.10, 2010.

Schumacher, F. X.; Hall, F. S. Logarithmic expression of timber-tree volume. Journal of Agricultural Research, v. 47, n. 9, p. 719-734, 1933.

SCHNEIDER, P. R. (1993) Introdução ao manejo florestal. Santa Maria: UFSM, CEPEF, FATEC. 
Scolforo, J. R. S. (2006) Biometria florestal: modelos de crescimento e produção florestal, LAVRAS: UFLA/FAEPE, pp. 393.

Vanclay, JK. (1994) Modelling forest growth and yield: applications to mixed tropical forests. Copenhagen: $\mathrm{CAB}$ International.

Volpato, E. Schmidt, P.B. Araujo, VC. (1972) - Carapa guianensis Aubl. (Andiroba). Estudos comparativos de tratamentos silviculturais. Acta amazônica, v. 2, n³ , p 7581. 